TRIAL WATCH

\title{
Dual-acting combination meets heart failure end point
}

Novartis has announced positive results from the Phase II PARAMOUNT trial of its first-in-class angiotensin receptor neprilysin inhibitor combination, LCZ696, in patients with heart failure with preserved ejection fraction (HFPEF). The dual-acting combination met its primary end point, causing a significantly greater reduction in levels of $\mathrm{N}$-terminal pro-brain natriuretic peptide (NT-proBNP), a $\mathrm{HF}$ marker, than treatment with an angiotensin receptor inhibitor alone (Lancet 26 Aug 2012: doi:10.1016/S0140-6736(12)61227-6).

HFPEF, which is characterized by inadequate myocardial relaxation and ventricular and vascular stiffness, constitutes half of all HF cases and the prevalence is rising. Several therapeutic options are available but their effects are limited and prognosis is poor. "Beta blockers and renin-angiotensin-aldosterone system (RAAS) inhibitors constitute the cornerstone for the treatment of HFPEF, but so far no treatment has been associated with consistent reductions in morbidity or mortality," says Jose López-Sendón, Hospital Universitario La Paz, Madrid, Spain. This is in contrast to HF with reduced ejection fraction (HFREF), in which the use of such agents has shown significant benefit. "Accordingly, treatment of HFPEF remains empirical and research to find new strategies is much needed," adds López-Sendón. Bertram Pitt, University of Michigan School of Medicine, USA, who is a consultant to Novartis, explains further: "One of the major challenges to therapy for HFPEF is the fact that patients are relatively older than those patients with HFREF and have a greater degree of comorbidity."
Uncertainty surrounding the pathophysiological mechanisms underlying HFPEF has hampered the development of novel therapies. "The pathophysiology of HFPEF remains controversial and therefore the targets to reduce cardiovascular mortality in patients are also controversial," says Pitt. "Concepts developed for HFREF have not translated into beneficial effects in HFPEF. It is uncertain whether this is just due to limited power or whether different mechanisms are involved," adds Karl Swedberg, University of Gothenburg, Sweden.

LCZ696 is composed of the approved angiotensin II type 1 receptor antagonist valsartan, which inhibits the vasoconstrictive action of angiotensin, and the novel neprilysin inhibitor prodrug AHU-377. Neprilysin is responsible for the degradation of atrial and B-type natriuretic peptides, which stimulate vasodilatation, diuresis and natriuresis and are released by the heart to defend from increased pressure and volume. "The effects of this molecule (LCZ696) are synergistic. It addresses the imbalance in vasoconstriction by direct vasodilatation and enhances the effects of the compensatory increased release of natriuretic peptides by reducing their breakdown," notes Swedberg. Although the idea of simultaneously targeting the RAAS and neprilysin is not new, previous strategies (such as omapatrilat) aimed to inhibit neprilysin and angiotensin-converting enzyme, which was associated with an increased risk of angioedema.

The PARAMOUNT study involved $301 \mathrm{HFPEF}$ patients with elevated levels of NT-proBNP (biologically inert and not a substrate for neprilysin), a marker of left ventricular stress.

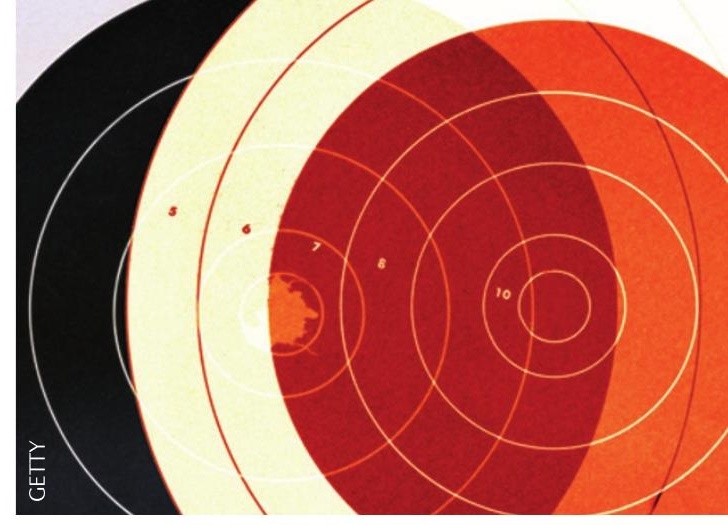

Patients received twice-daily treatment with either LCZ696 titrated to $200 \mathrm{mg}$ or valsartan titrated to $160 \mathrm{mg}$ for 36 weeks. After 12 weeks, the reduction in NT-proBNP was $23 \%$ greater with LCZ696 than valsartan (reductions in NT-proBNP have been associated with improved outcomes), although the difference was not significant by 36 weeks. In addition, there was a greater reduction in left atrial size in LCZ696-treated patients at the end of the 36-week study (an enlarged left atrium is characteristic of HF). Importantly, LCZ696 had an acceptable safety profile and was well tolerated.

"These findings suggest a beneficial effect of this agent on myocardial diastolic function and therefore cardiovascular outcomes in patients with HFPEF," says Pitt. "However, the ultimate role of LCZ696 in HFPEF therapy will be determined by further large-scale prospective randomized trials," he adds.

The data are certainly promising, although there are several limitations of the Phase II study. "The diagnosis of HFPEF is elusive and may include patients with different comorbidities. In addition, the number of cases included in the trial was relatively small, HF was moderate and the reduction in NT-proBNP was transient. Most importantly, the reduced number of cases and the length of the follow-up do not permit to ascertain long-term safety," highlights López-Sendón. “In any case, LCZ696 is a new, promising drug for the treatment of patients with HFPEF, and its possible impact on clinical outcomes should be explored in a large outcomes clinical trial," he concludes.

Sarah Crunkhorn 